\title{
Association of MSH6 mutation with glioma susceptibility, drug resistance and progression (Review)
}

\author{
CHAORAN XIE ${ }^{1,2^{*}}$, HANSONG SHENG $^{2 *}$, NU ZHANG $^{2}$, SHITING LI $^{1}$, XIANGYU WEI ${ }^{1}$ and XUESHENG ZHENG ${ }^{1}$ \\ ${ }^{1}$ Department of Neurosurgery, Xinhua Hospital Affiliated to Shanghai Jiao Tong University School of Medicine, \\ Shanghai 200092; ${ }^{2}$ Department of Neurosurgery, The Second Affiliated Hospital of \\ Wenzhou Medical University, Wenzhou, Zhejiang 325027, P.R. China
}

Received February 12, 2016; Accepted May 9, 2016

DOI: $10.3892 / \operatorname{mco} .2016 .907$

\begin{abstract}
MutS homolog 6 (MSH6) is one of the mismatch repair proteins and is encoded by the MSH6 gene, which is located on chromosome 2 and is 23,806 bp in length, including 10 exons and 83 untranslated regions. The MSH6 protein consists of 1,358 amino acid residues and forms a heterodimer with another mismatch repair protein, MSH2. The MSH2-MSH6 heterodimeric complex is able to recognize base-base substitution and single-base insertion/deletion mismatches. Germline mutations of MSH6 lead to high susceptibility to glioma, as well as a number of benign or malignant tumors in other organs. However, somatic MSH6 mutations are not associated with susceptibility to glioma. Somatic MSH6 mutations usually follow temozolomide treatment and result in resistance to temozolomide. Subsequently, MSH6 mutations cause a hypermutation in the glioma cell genome, which may accelerate tumor progression.
\end{abstract}

\section{Contents}

1. Introduction

2. Genetic location and function of MSH6

3. Germline MSH6 mutations and cancer susceptibility

4. Somatic MSH6 mutations, glioma recurrence and drug resistance

5. MSH6 mutations lead to tumor genome hypermutation

Correspondence to: Dr Xuesheng Zheng, Department of Neurosurgery, Xinhua Hospital Affiliated to Shanghai Jiao Tong University School of Medicine, 1665 Kongjiang Road, Yangpu, Shanghai 200092, P.R. China

E-mail: pheiphei@126.com

*Contributed equally

Key words: mutS homolog 6, mutation, glioma, drug resistance, temozolomide

\section{Introduction}

Glioma is the most frequent malignant brain tumor in adults. This tumor is characterized by diffuse infiltration of the brain and frequent local recurrence. A malignant tumor is considered to be the result of an accumulation of gene mutations, and tumor progression is driven by additional mutations (1). DNA is constantly exposed to biological, physical and chemical damage that may result in gene mutations; the DNA mismatch repair (MMR) system is responsible for repairing DNA damage. As a result, gene mutations are significantly more frequent in MMR-deficient cells $(2,3)$. Theoretically, mutations in the MMR system may cause additional mutations in numerous other genes, initiating a cascade of mutations throughout the cancer genome. Some of these mutations may confer selective advantages, which enable the mutated cells to proliferate and achieve clonal dominance (4). Growing evidence indicates that a deficiency of MMR genes is associated with the recurrence and drug resistance of glioma. Candidate genes of interest include mutL homolog (MLH)1, mutS homolog (MSH)2 and MSH6 (4,5). In particular, MSH6 mutations are considered to play an important role in the recurrence of glioma, acquired resistance to alkylating agents and genome instability $(3,6,7)$. The aim of the present study was to review the association between MSH6 mutations and glioma.

\section{Genetic location and function of MSH6}

Mismatches inevitably occur during DNA replication. Prokaryotic as well as eukaryotic organisms are equipped with enzymatic systems to repair these mismatches. The bacterial MutHLS system repairs single-base mismatches and small insertion/deletions. Eukaryotic cells possess respective counterpart enzymes to repair mismatches. In yeast, mammalian cells and other organisms, the MMR systems are similar to the prokaryotic MutHLS system, and are therefore named MSHs and MLHs (8). Certain MMR proteins have unique functions (9). MMR proteins initiate the repair of mismatches via the following steps: Recognition of the mismatch, assembly of the repairosome, degradation of the error-containing DNA strand and, finally, repair synthesis (10). 
The MSH6 protein is one of the MMR proteins and is also referred to as G/T-binding protein or p160 (8). The highest level of MSH6 expression has been detected in the testis (8). The human MSH6 gene is located on chromosome 2 and is 23,806 bp in length, including 10 exons and 83 untranslated regions. The MSH6 protein consists of 1,358 amino acid residues (8). Transcription of MSH6 increases during the late G1 phase. In humans, transcription is increased $\sim 2.5$-fold during the late G1/early $\mathrm{S}$ phase. However, the protein level remains almost invariant throughout the cell cycle; since MSH6 binds to MSH2 and MSH2 is expressed in a cell cycle-independent manner, any free MSH6 protein is degraded (10). The MSH6 protein comprises five regions: A mismatch binding domain, a connector region, two $\alpha$-helical regions separated by a clamp region and adenosine triphosphate (ATP) binding cassette-ATPase. MSH6 also contains another domain of 340 amino acids, which is not related to MMR activity (11).

The MSH6 gene may be transcribed from a housekeeping gene promoter $(10,12)$. The MSH6 gene contains no TATAA- or CAAT-boxes in its translation initiation site, which is surrounded by sequences containing $\mathrm{CpG}$ islands. There are multiple start sites for transcription and copies of consensus-binding sequences of the transcription factors, including specificity protein $(\mathrm{Sp}) 1, \mathrm{ETF}$, meta-regulatory transcription factor-1 and activator protein-1. The gene contains seven functional Sp1 transcription-factor binding sites in the 5'-flanking region, which bind $\mathrm{Spl}$ and $\mathrm{Sp} 3$ and regulate MSH6 promoter activity (12).

MSH2, MSH3 and MSH6 are the main molecules responsible for mismatch recognition in eukaryotic cells and they form two heterodimeric complexes: MSH2-MSH6 and MSH2-MSH3. The MSH2-MSH6 complex is able to recognize base-base substitution and single-base insertion/deletion mismatches. After recognition of the error, the MSH2-MSH6 complex binds to the mismatch and forms a ring around the DNA, which moves along the DNA chain and repairs it (9). The complex maintains genomic integrity and inhibits the generation of tumors by means of repairing errors occurring during replication $(13,14)$. The MMR function of MSH2-MSH6 is inhibited by cadmium (15). The MSH2-MSH3 complex can only repair insertion/deletion mismatches and mostly repairs larger mismatches; therefore, it cannot fully compensate for the function of the MSH2-MSH6 complex if the MSH6 gene is mutant (8).

\section{Germline MSH6 mutations and cancer susceptibility}

Since germline MSH6 mutations affect all organs, the effect of MMR deficiency is extensive, and the resulting cancer susceptibility is diffuse, including the central nervous system.

Edelmann et al (8) investigated cancer susceptibility in mice with MSH6 mutation produced by a gene-targeting vector. Neither the transcript nor the protein of MSH6 were detectable in the cells of mice carrying a homozygous mutation. The viability of mice with MSH6 mutation was obviously reduced and their lifespan was also shortened. The mice developed lymphomas, gastrointestinal tumors and other types of tumors in the liver, lung, skin and soft tissues.

Regarding the mechanism of MSH6 mutations causing tumors, two theories are prevalent: According to one theory, an accumulation of mutations in suppressor genes and proto-oncogenes occurs due to loss of the repair function. Another mechanism may involve the loss of apoptotic response to DNA damage. This deficiency in the apoptotic response to DNA damage may lead to the resistance of tumors to chemotherapy (9). In certain tumor cell lines, the emergence of MSH6 mutations led to resistance to alkylating agents (8).

Compared to individuals with wild-type MSH6, carriers of germline mutations of MSH6 have a significantly increased risk of developing cancer. For example, analysis of 113 families of MSH6 mutation carriers revealed that carriers have an 8-fold increased incidence of colorectal cancer and a 26-fold increased incidence of endometrial cancer (16). Germline mutation of MSH6 leads to hereditary non-polyposis colon cancer (HNPCC), also referred to as Lynch syndrome. Such patients are also more susceptible to a wide spectrum of tumors, even more than to colon cancer, including tumors of the rectum, endometrium, small bowel, urinary tract and brain (17,18). Unlike early-onset HNPCC driven by MSH2 and MLH1 mutations, familial HNPCC driven by MSH6 mutations is associated with a later onset $(9,10,12,19)$.

A previous study reported on two brothers with MSH6 loss who presented with $>1$ tumors: One patient was diagnosed with non-Hodgkin lymphoma and anaplastic oligodendroglioma, while the other patient had medulloblastoma (20). In another case study, the offspring of consanguineous healthy parents was diagnosed with neurofibromatosis type 1, oligodendroglioma and rectal cancer; a homozygous c.3386_3388delGTG mutation in exon 5 of the MSH6 gene was identified (21).

In 19 members of four unrelated Ashkenazi families, a common c.3984_3987dup mutation of the MSH6 gene was detected, which led to development of HNPCC (22). In Chinese HNPCC family studies, three novel missense mutations and a novel single-nucleotide polymorphism were identified, both of which may have caused HNPCC (17).

Biallelic germline MSH6 mutations may lead to constitutional MMR-deficiency syndrome (CMMR-D); this condition differs from Lynch syndrome regarding its mode of inheritance. CMMR-D is a rare autosomal recessive disease and was found to be associated with the onset of brain tumors, hematological malignancies and tumors of the large intestine at an early age (2). Glioblastomas, astrocytomas and oligodendrogliomas frequently occur in patients with CMMR-D $(23,24)$.

In a Romani family, three individuals carried biallelic c.3261insC (p.F1088LfsX5) mutations of MSH6 and were diagnosed with brain gliomas and 'café-au-lait' hyperpigmentations. Two of the patients were siblings, and the two pairs of parents were carriers of heterozygous MSH6 mutations, without any cancers (23). Ripperger et al (25) reported on a female patient diagnosed with T-cell non-Hodgkin lymphoma at the age of 6 years and with colonic adenocarcinoma with local lymph node metastases at the age of 13 years, who carried a novel biallelic MSH6 mutation.

A homozygous germline mutation of MSH6 was detected in two Pakistani siblings, who were diagnosed with Turcot syndrome (association of central nervous system malignant tumors and familial colorectal cancer). Both patients displayed characteristics of neurofibromatosis type 1; the male sibling was diagnosed with lymphoma at the age of 5 years and colorectal cancer at the age of 8 years, while the female sibling was 
diagnosed with glioblastoma multiforme (GBM) at the age of 8 years. In the female patient, a novel homozygous single-base insertion mutation was found by sequencing a blood sample. The mutation (3634insT) caused a premature stop at codon 1,223 in exon 7. The parents of the two siblings carried heterozygous 3634insT mutations of MSH6, but had no cancer (26).

\section{Somatic MSH6 mutations, glioma recurrence and drug resistance}

Gliomas are malignancies derived from neuroepithelial tissue; they account for half of all diagnosed primary brain tumors and have a poor prognosis. High-grade gliomas (HGGs) include anaplastic astrocytoma and GBM. While certain HGGs are primary malignant tumors, others are secondary HGGs arising from malignant transformation of low-grade gliomas (23). The current standard of care for glioma is surgical resection followed by adjuvant radiotherapy (XRT) and administration of the alkylating agent temozolomide (TMZ). It has been demonstrated that this common regimen increases the overall and disease-free survival of patients with glioblastomas $(6,7,11)$. However, the median survival is only 14.6 months (11). Furthermore, almost all patients with HGG suffer a relapse and eventually develop resistance to alkylating agents $(6,7)$.

The mechanism of action of TMZ in gliomas is as follows (11): TMZ is degraded to 5-(3-methyl-1-triazeno) imidazole-4-carboxamide and then to methylhydrazine, which then effectively methylates DNA to generate three main adducts, N7-methylguanine (70\%), N3-methyladenine (9\%) and O6-methylguanine (O6-me-G; 6\%) (27). The generation of O6-me-G is the main cause of the cytotoxic effects of TMZ. During replication, O6-me-G is incorrectly paired with thymine. The MMR system can recognize the mismatch of O6-me-G/T coupling and subsequently activate the apoptotic response. The DNA repair protein O6-me-G-DNA alkyltransferase (MGMT; also referred to as AGT), removes the O6-methyl adduct from guanine, which decreases the toxicity of TMZ. Particularly in human brain tumors, the activity of MGMT may increase up to $\sim 300$-fold (27). Thus, MGMT counteracts the therapeutic effect of TMZ and is the major cause of resistance to TMZ, accounting for $\sim 70-80 \%$ of cases with resistance to alkylating agents. However, methylation of the MGMT gene promoter silences its expression. Patients with a methylated MGMT promoter region benefit more from TMZ treatment compared with patients with an unmethylated promoter $(5,28)$. MGMT-methylated cases have longer progression-free survival (PFS), overall survival (OS) and post-recurrence survival (PRS) (28). However, the methylation status of MGMT does not change during TMZ treatment $(11,28)$. Therefore, in glioma patients with a methylated MGMT promoter who are initially sensitive to TMZ, but become resistant after TMZ chemotherapy, the resistance is mediated by other mechanisms (11). This alternative mechanism involves inactivation of MSH6, which was found to be responsible for TMZ resistance of glioblastomas following TMZ chemotherapy (29,30). Cahill et al (30) compared MGMT staining, MSH6 mutation status and protein expression in glioblastomas pre- and post-treatment. The MGMT status in the tumor following XRT+TMZ treatment was identical to that prior to treatment in each patient, leading to the conclusion that the development of secondary resistance to TMZ after treatment cannot be attributed to MGMT. Of note, no MSH6 mutations were present in the glioma samples prior to treatment, while they were detected in certain patients following XRT+TMZ treatment. While none of the patients were treated with TMZ without XRT, in accordance with clinical guidelines, there were no post-TMZ-alone specimens and MSH6 protein expression was not affected by XRT alone; however, MSH6 protein loss was observed following XRT+TMZ treatment. These results indicated that mutations of MSH6, but not of MGMT, are accountable for acquired TMZ resistance in glioma patients (30).

Hunter et al (6) performed sequencing analyses of the MSH6 gene in 9 cases of malignant gliomas. Somatic MSH6 mutations were found in 2 recurrent glioblastoma cases (PD1487a and PD1489a) with a history of XRT+TMZ treatment, while mutations were not present in the primary tumors of these patients. MSH6 mutations were not found in any cases prior to treatment, indicating that somatic MSH6 mutations are not responsible for the pathogenesis of glioma. The authors concluded that somatic MSH6 mutations result from TMZ treatment and hypothesized that they may lead to the survival of the affected glioma cell and its clones, finally leading to a relapse.

A subsequent study further assessed MSH6 gene mutations and protein expression in glioblastomas prior to and following treatment (7). XRT alone did not affect the MSH6 mutation status or protein expression levels; however, in $\sim 50 \%$ of recurrent gliomas following TMZ+XRT treatment, MSH6 mutations and reduced MSH6 protein expression were detected. Genomic analysis of 91 glioblastomas and their matched peripheral blood samples was performed in a Cancer Genome Atlas (TCGA) study (3). Of 19 recurrent glioblastoma cases treated with alkylating agents, 5 patients $(26 \%)$ carried non-synonymous MSH6 mutations. Furthermore, sequencing analysis of matched pre- and post-treatment unstained formalin-fixed, paraffin-embedded tumor tissue sections from 4 of the 5 non-synonymous MSH6 mutation cases revealed that MSH6 mutation was absent in pretreatment glioblastomas, strongly suggesting that the MSH6 mutation was a consequence of TMZ treatment (3).

However, there are certain exceptions: A recent study reported the presence of MSH6 mutations in anaplastic oligodendroglioma and pretreatment glioblastoma, and that these mutations may affect resistance to TMZ, independent of the MGMT promoter methylation status. MSH6 mutations may arise in GBM prior to alkylating therapy, as well as in glioma subtypes other than GBM (31). Considering that such exceptions are rare, they should be considered as accidental mutations. Thus, it may be concluded that the majority of somatic MSH6 mutations are caused by TMZ.

Since MGMT expression is mainly regulated by promoter methylation, it is worth investigating whether MSH6 protein levels are also controlled by methylation. Felsberg et al (28) found that the methylation status of MSH6 did not change in 43 paired primary and recurrent glioblastoma specimens. However, the expression of MSH6 was significantly lower in recurrent cases compared with that in primary cases, suggesting that the loss of MSH6 protein does not result from 
promoter methylation but via other mechanisms. In accordance with these results, Rodriguez-Hernandez et al (13) reported no association between protein expression and the promoter methylation status of MSH6.

To confirm whether the loss of MSH6 occurs at the transcriptional level, its mRNA levels were measured by polymerase chain reaction analysis. The results revealed that various amounts of mRNA were detectable in all samples regardless of MSH6 protein loss, suggesting that mutated MSH6 genes were transcribed but the protein levels were either below the limit of detection of the immunofluorescence staining or the protein structure was altered, becoming unrecognizable by the antibodies (11).

Several experiments have been performed to investigate the effects of MSH6 mutations on the resistance of glioma to TMZ (7). The A172 glioblastoma cell line was treated with TMZ for 3 weeks and the exposure to TMZ generated a highly resistant cell line, A172TR3, in which MSH6 protein expression was significantly downregulated. Sequencing analysis of the A172TR3 cell line revealed the c.3656C $>$ T somatic MSH6 mutation, which results in Thr1219Ile. The survival of A172TR3 in the presence of TMZ was significantly longer compared with that of the A172 cell line. However, the growth rates of the two cell lines were similar in culture without TMZ. In another experiment, the U251 glioblastoma cell line was transfected with lentiviral particles generating small hairpin (sh)RNA mediating MSH6 knockdown, which increased the cells' resistance to TMZ compared with that of the parental cell line U251. Conversely, after reconstitution of MSH6 expression in the Gli60 glioblastoma cell line, which carries the MSH6 mutation delG2425, by using a lentiviral overexpression vector, the sensitivity to TMZ was increased. These results support the association of MSH6 mutations with resistance of glioblastomas to TMZ (7). Sensitivity to $\mathrm{TMZ}$ is indicated to require the absence of MGMT as well as the presence of functional MMR (31). O6-benzylguanine (O6-BG) and O6-(4-bromothenyl) guanine may function as pseudosubstrates to inhibit the function of MGMT $(27,31)$. In the abovementioned studies, O6-BG was used to alleviate the effect of MGMT when necessary.

Nguyen et al (31) investigated the differential sensitivity of glioma cells to TMZ depending on their methylation and mutation status (MGMT-methylated/MSH6 wild-type vs. MGMT-unmethylated/MSH6 wild-type vs. MGMTmethylated/MSH6 mutant). All MGMT-methylated/MSH6 wild-type brain tumor cells exhibited a significant response to TMZ. However, MGMT-methylated/MSH6-mutant glioma cells were less sensitive to TMZ and MGMT-unmethylated cells were insensitive to TMZ regardless of their MSH6 mutation status. The survival time of mice xenografted with the MGMT-methylated/MSH6 wild-type cell line was distinctly increased by TMZ treatment, while TMZ did not significantly affect the median survival of those xenografted with the MGMT-unmethylated/MSH6 wild-type cell line and only modestly increased the survival of mice inoculated with the MGMT-methylated/MSH6-mutant cell line.

By contrast, Maxwell et al (11) stated that MSH6 mutations do not confer clinical resistance to TMZ in malignant glioma, based on the finding that MSH6 mutation or loss of MSH6 protein expression is not associated with high microsatellite instability
(MSI). MSI is a state of genetic hypermutability resulting from impaired DNA MMR. Microsatellites are repeated DNA sequences comprising repeating units of 1-6 base pairs in length. Despite the high inter-individual variability of microsatellites, contributing to the individual DNA 'fingerprint', microsatellite length is constant within each individual. In cells with dysfunctional MMR, errors occurring during DNA replication cannot be corrected, consequently leading to an accumulation of errors and the generation of novel microsatellites fragments, which results in MSI. For the identification of MSI, the five quasimonomorphic mononucleotide loci, including BAT-25 (c-kit), BAT-26 (hMSH2), NR-21 (SLC7A8), NR-22 (IMP1) and NR-24 (ZNF-2) must be detected (11). MSI-high is defined as $>3 / 5$ unstable loci and MSI-low is defined as $<2 / 5$ unstable loci. Single-locus MSI was observed in only 3\% of glioma patient samples with MSH6 loss, while no sample was classified as MSI-high. This result indicated that the expression of MSH6 was not associated with MSI (11), which has been confirmed by other studies $(5,7,9)$. The inactivation of MSH6 is only associated with the MSI-low phenotype, but not with MSI-high (7). However, the finding that MSH6 mutations are not associated with MSI-high does not lead to the conclusion that MSH6 mutations do not mediate TMZ resistance. Maxwell et al (11) did not compare directly the cytotoxic effect of TMZ in gliomas with or without MSH6 mutation; thus, their conclusion is not supported by direct evidence.

\section{MSH6 mutations lead to tumor-genome hypermutation}

Tumors are derived from chromosomal aberrations, nucleotide substitutions and epigenetic modifications (3). Tumors develop via clone selection of cells with growth advantages arising from genetic alterations. During cancer progression, mutants and their clones overcome a series of selection factors and acquire increasingly malignant characteristics. Similarly, gliomas may accumulate genetic alterations in oncogenes and tumor suppressor genes that confer a selective advantages under treatments (30).

Hunter et al (6) reported that in two patients (PD1487a and PD1489a) carrying a MSH6 mutation, a large number of mutations were present in the tumor genome (34 and 30 mutations, respectively, within the sequenced 500-kb DNA, accounting for $\sim 1$ mutation per $15 \mathrm{~kb}$ ). In total, these two glioma samples had an estimate of $>200,000$ somatic point mutations in their genomes; assuming a random distribution, there are $\sim 1,500$ amino acid changes in each tumor, affecting $\sim 8 \%$ of protein coding. This phenomenon, referred to as hypermutation, facilitates rapid evolution of clones with growth advantages and tumor progression. The mutation patterns in the 2 cases were highly similar: They were all single-base substitutions and most substitutions were $\mathrm{C}: \mathrm{G}>\mathrm{T}:$ A mutations $(63 / 64 ; 98 \%)$, which were found in a specific context. $\mathrm{C}: \mathrm{G}>\mathrm{T}$ :A mutations of this type mostly occur at $\mathrm{CpC}$ dinucleotides where the $5^{\prime}$ cytosine is mutated (6). This pattern is different from the common $\mathrm{C}: \mathrm{G}>\mathrm{T}$ :A mutation caused by deamination of 5-methylcytosine, which occurs at CpG dinucleotides.

A similar finding was reported by Yip et al (7): A high rate of $\mathrm{C}: \mathrm{G}>\mathrm{T}$ :A mutations was present at $\mathrm{CpC}$ dinucleotides in 4 cases of MSH6-mutant glioblastoma. 
According to a TCGA study analyzing 91 gliomas, MSH6 mutations in treated gliomas lead to a preponderance of G:C $>$ A:T transitions at non-CpG sites (81\%) (3), which was confirmed by a more recent study, particularly regarding transitions during TMZ treatment (15).

In conclusion, if the MSH6 mutation are present in glioma, TMZ is not only ineffective, but may also act as a catalyst to tumor progression. If reconstituting a fully functional MMR system is not feasible, MSH6 mutation should be identified as early as possible, so as to adjust the chemotherapy accordingly.

\section{References}

1. Loeb LA, Loeb KR and Anderson JP: Multiple mutations and cancer. Proc Natl Acad Sci USA 100: 776-781, 2003.

2. Bougeard G, Olivier-Faivre L, Baert-Desurmont S, Tinat J, Martin C, Bouvignies E, Vasseur S, Huet F, Couillault G, Vabres P, et al: Diversity of the clinical presentation of the MMR gene biallelic mutations. Fam Cancer 13: 131-135, 2014

3. Cancer Genome Atlas Research Network: Comprehensive genomic characterization defines human glioblastoma genes and core pathways. Nature 455: 1061-1068, 2008.

4. Stark AM, Doukas A, Hugo HH, Hedderich J, Hattermann K, Maximilian Mehdorn $\mathrm{H}$ and Held-Feindt J: Expression of DNA mismatch repair proteins MLH1, MSH2, and MSH6 in recurrent glioblastoma. Neurol Res 37: 95-105, 2015.

5. Stark AM, Doukas A, Hugo HH and Mehdorn HM: The expression of mismatch repair proteins MLH1, MSH2 and MSH6 correlates with the Ki67 proliferation index and survival in patients with recurrent glioblastoma. Neurol Res 32: 816-820, 2010.

6. Hunter C, Smith R, Cahill DP, Stephens P, Stevens C, Teague J, Greenman C, Edkins S, Bignell G, Davies H, et al: A hypermutation phenotype and somatic MSH6 mutations in recurrent human malignant gliomas after alkylator chemotherapy. Cancer Res 66: 3987-3991, 2006

7. Yip S, Miao J, Cahill DP, Iafrate AJ, Aldape K, Nutt CL and Louis DN: MSH6 mutations arise in glioblastomas during temozolomide therapy and mediate temozolomide resistance. Clin Cancer Res 15: 4622-4629, 2009.

8. Edelmann W, Yang K, Umar A, Heyer J, Lau K, Fan K, Liedtke W, Cohen PE, Kane MF, Lipford JR, et al: Mutation in the mismatch repair gene Msh6 causes cancer susceptibility. Cell 91: 467-477, 1997.

9. Yang G, Scherer SJ, Shell SS, Yang K, Kim M, Lipkin M, Kucherlapati R, Kolodner RD and Edelmann W: Dominant effects of an Msh6 missense mutation on DNA repair and cancer susceptibility. Cancer Cell 6: 139-150, 2004.

10. Szadkowski $M$ and Jiricny J: Identification and functional characterization of the promoter region of the human MSH6 gene. Genes Chromosomes Cancer 33: 36-46, 2002.

11. Maxwell JA, Johnson SP, McLendon RE, Lister DW, Horne KS, Rasheed A, Quinn JA, Ali-Osman F, Friedman AH, Modrich PL, et al: Mismatch repair deficiency does not mediate clinical resistance to temozolomide in malignant glioma. Clin Cancer Res 14: 4859-4868, 2008.

12. Gazzoli I and Kolodner RD: Regulation of the human MSH6 gene by the Sp1 transcription factor and alteration of promoter activity and expression by polymorphisms. Mol Cell Biol 23: 7992-8007, 2003.

13. Rodriguez-Hernández I, Garcia JL, Santos-Briz A, Hernández-Laín A, González-Valero JM, Gómez-Moreta JA, Toldos-González O, Cruz JJ, Martin-Vallejo J and González-Sarmiento R: Integrated analysis of mismatch repair system in malignant astrocytomas. PLoS One 8: e76401, 2013.

14. Slean MM, Panigrahi GB, Ranum LP and Pearson CE: Mutagenic roles of DNA 'repair' proteins in antibody diversity and disease-associated trinucleotide repeat instability. DNA Repair (Amst) 7: 1135-1154, 2008

15. Wieland M, Levin MK, Hingorani KS, Biro FN and Hingorani MM: Mechanism of cadmium-mediated inhibition of Msh2-Msh6 function in DNA mismatch repair. Biochemistry 48: 9492-9502, 2009.
16. Baglietto L, Lindor NM, Dowty JG, White DM, Wagner A, Gomez Garcia EB, Vriends AH; Dutch Lynch Syndrome Study Group, Cartwright NR, Barnetson RA, et al: Risks of Lynch syndrome cancers for MSH6 mutation carriers. J Natl Cancer Inst 102: 193-201, 2010

17. Yan SY, Zhou XY, Du X, Zhang TM, Lu YM, Cai SJ, Xu XL, Yu BH, Zhou HH and Shi DR: Three novel missense germline mutations in different exons of MSH6 gene in Chinese hereditary non-polyposis colorectal cancer families. World J Gastroenterol 13: 5021-5024, 2007.

18. Zhao YS, Hu FL, Wang F, Han B, Li DD, Li XW and Zhu S: Meta-analysis of MSH6 gene mutation frequency in colorectal and endometrial cancers. J Toxicol Environ Health A 72: 690-697, 2009

19. Verma L, Kane MF, Brassett C, Schmeits J, Evans DG, Kolodner RD and Maher ER: Mononucleotide microsatellite instability and germline MSH6 mutation analysis in early onset colorectal cancer. J Med Genet 36: 678-682, 1999.

20. Poley JW, Wagner A, Hoogmans MM, Menko FH, Tops C, Kros JM, Reddingius RE, Meijers-Heijboer H, Kuipers EJ and Dinjens WN; Rotterdam Initiative on Gastrointestinal Hereditary Tumors: Biallelic germline mutations of mismatch-repair genes: A possible cause for multiple pediatric malignancies. Cancer 109: 2349-2356, 2007.

21. Menko FH, Kaspers GL, Meijer GA, Claes K, van Hagen JM and Gille JJ: A homozygous MSH6 mutation in a child with café-au-lait spots, oligodendroglioma and rectal cancer. Fam Cancer 3: 123-127, 2004

22. Goldberg Y, Porat RM, Kedar I, Shochat C, Galinsky D, Hamburger T, Hubert A, Strul H, Kariiv R, Ben-Avi L, et al: An Ashkenazi founder mutation in the MSH6 gene leading to HNPCC. Fam Cancer 9: 141-150, 2010.

23. Ilencikova D, Sejnova D, Jindrova J and Babal P: High-grade brain tumors in siblings with biallelic MSH6 mutations. Pediatr Blood Cancer 57: 1067-1070, 2011.

24. Therkildsen C, Ladelund S, Rambech E, Persson A, Petersen A and Nilbert M: Glioblastomas, astrocytomas and oligodendrogliomas linked to Lynch syndrome. Eur J Neurol 22: 717-724, 2015.

25. Ripperger T, Beger C, Rahner N, Sykora KW, Bockmeyer CL, Lehmann U, Kreipe $\mathrm{HH}$ and Schlegelberger B: Constitutional mismatch repair deficiency and childhood leukemia/lymphoma-report on a novel biallelic MSH6 mutation. Haematologica 95: 841-844, 2010.

26. Hegde MR, Chong B, Blazo ME, Chin LH, Ward PA, Chintagumpala MM, Kim JY, Plon SE and Richards CS: A homozygous mutation in MSH6 causes Turcot syndrome. Clin Cancer Res 11: 4689-4693, 2005.

27. Zhang J, Stevens MF, Laughton CA, Madhusudan S and Bradshaw TD: Acquired resistance to temozolomide in glioma cell lines: Molecular mechanisms and potential translational applications. Oncology 78: 103-114, 2010.

28. Felsberg J, Thon N, Eigenbrod S, Hentschel B, Sabel MC, Westphal M, Schackert G, Kreth FW, Pietsch T, Löffler M, et al: Promoter methylation and expression of MGMT and the DNA mismatch repair genes MLH1, MSH2, MSH6 and PMS2 in paired primary and recurrent glioblastomas. Int J Cancer 129: 659-670, 2011

29. Cahill DP, Codd PJ, Batchelor TT, Curry WT and Louis DN: MSH6 inactivation and emergent temozolomide resistance in human glioblastomas. Clin Neurosurg 55: 165-171, 2008.

30. Cahill DP, Levine KK, Betensky RA, Codd PJ, Romany CA, Reavie LB, Batchelor TT, Futreal PA, Stratton MR, Curry WT, et al: Loss of the mismatch repair protein MSH6 in human glioblastomas is associated with tumor progression during temozolomide treatment. Clin Cancer Res 13: 2038-2045, 2007.

31. Nguyen SA, Stechishin OD, Luchman HA, Lun XQ, Senger DL, Robbins SM, Cairncross JG and Weiss S: Novel MSH6 mutations in treatment-naïve glioblastoma and anaplastic oligodendroglioma contribute to temozolomide resistance independently of MGMT promoter methylation. Clin Cancer Res 20: 4894-4903, 2014. 